\title{
RELAÇÃO ENTRE A TENSÃO APLICADA E A SENSAÇÃO DE DESCONFORTO NOS MÚSCULOS ISQUIOTIBIAIS DURANTE O ALONGAMENTO
}

\author{
Branco VR ${ }^{2}$, Negrão Filho RF ${ }^{1}$, Padovani $\mathrm{CR}^{3}$, Azevedo FM ${ }^{4}$, Alves $\mathrm{N}^{5}$ e Carvalho AC ${ }^{1}$ \\ ${ }^{1}$ Departamento de Fisioterapia, Faculdade de Ciência e Tecnologia, Universidade Estadual Paulista Júlio de Mesquita \\ Filho - UNESP, Presidente Prudente, SP - Brasil \\ ${ }^{2}$ Fisioterapeuta \\ ${ }^{3}$ Departamento de Bioestatística, Instituto de Biociências, UNESP, Botucatu, SP - Brasil \\ ${ }^{4}$ Laboratório de Fisioterapia Aplicada ao Movimento Humano, Departamento de Fisioterapia, Faculdade de Ciência e \\ Tecnologia, UNESP, Presidente Prudente, SP - Brasil \\ ${ }^{5}$ Departamento de Física, Química e Biologia, Faculdade de Ciência e Tecnologia, UNESP, Presidente Prudente, SP - \\ Brasil \\ Correspondência para: Rúben de Faria Negrão Filho, Departamento de Fisioterapia da FCT, UNESP, Rua Roberto \\ Simonsen, 305, CEP 19060-900 Presidente Prudente, SP - Brasil
}

Recebido: 06/05/2005 - Revisado: 20/03/2006 - Aceito: 13/07/2006

\begin{abstract}
RESUMO
Contextualização: O alongamento muscular é amplamente empregado no tratamento e na prevenção de doenças musculoesqueléticas. Estudos utilizam informações subjetivas do paciente como “desconforto" e "tensão sem dor” para limitar intensidade de alongamento, sem estabelecer uma tensão adequada de estiramento. Objetivo: Estudar a relação entre tensão aplicada e informações subjetivas, durante alongamento estático passivo para analise da reprodutibilidade e variações nas informações sensoriais. Métodos: Participaram 20 jovens com idade média de 20 anos $( \pm 2,25)$, sem experiência prévia com programa de alongamento, divididos em dois grupos: G1 - participou de programa de alongamento dos músculos isquiotibiais, G2 - sem programa de alongamento. Os grupos foram submetidos a três avaliações para mensurar o torque e a atividade eletromiográfica (EMG), nas posições de sensação de desconforto (SD) sem dor e sensação de desconforto com dor (SDD), por meio de um sistema de aquisição de sinais, constituído de condicionador de sinais, eletrodo bipolar ativo de superfície, célula de carga, eletrogoniômetro, sensor de pressão e modelo biomecânico. Resultado: As análises do torque nas posições SD e SDD revelaram comportamento semelhante nos dois grupos, sem diferenças significantes entre as três avaliações (P > 0.05), demonstrando reprodutibilidade das informações subjetivas dos grupos nas duas posições. A EMG nas posições de SD e SDD não mostrou diferença significante com a EMG obtida em repouso. Conclusão: Este trabalho revelou que a informação da sensação subjetiva de alongamento é confiável, segura e possível de ser reproduzida clinicamente.
\end{abstract}

Palavras-chave: alongamento, tensão, torque, eletromiografia.

\section{ABSTRACT \\ Relationship Between Tension Applied and Sensations of Discomfort in the Hamstring Muscle During Stretching}

Background: Muscle stretching is widely used in the prevention and treatment of musculoskeletal diseases. Previous studies have used subjective information like "discomfort" and "tension without pain" for limiting the intensity of stretching, but without establishing appropriate tensions for stretching. Objective: To study the relationship between applied tension and subjective information during static passive stretching, in order to analyze the reproducibility and variability of sensorial information. Method: Twenty subjects (mean age: $20 \pm 2.25$ years) without previous experience of stretching programs were recruited and divided into two groups. G1 participated in a hamstring muscle stretching program, while G2 did not follow a stretching program. Both groups underwent three evaluations to quantify torque and electromyographic activity, in positions that produced a sensation of discomfort without pain (SD) and a sensation of discomfort with pain (SDP), using a signal acquisition system consisting of a signal conditioner, active bipolar surface electrode, load cell, electrogoniometer, pressure sensor and biomechanical model. Results: The torque analyses in the SD and SDP positions showed similar behavior in the two groups, without significant 
differences between the three evaluations ( $p>0.05$ ), thereby demonstrating the reproducibility of the subjective information from the groups in the two positions. Electromyography in the SD and SDP positions did not show significant differences in relation to the findings at rest. Conclusion: This study demonstrated that the subjective information on stretching sensations is reliable and secure, and can be reproduced clinically.

Key words: stretching, tension, torque, electromyography.

\section{INTRODUÇÃO}

Dentre os diversos recursos empregados pelos fisioterapeutas no tratamento e prevenção de doenças musculoesqueléticas, destaca-se o alongamento muscular, por ser largamente utilizado. No entanto, ainda não há um consenso de como executá-lo de forma a garantir sua máxima eficiência. Isso se deve às dúvidas geradas a partir da diversidade de técnicas, número de repetições, freqüência, tempo de duração e, principalmente, sobre a intensidade de tensão que deve ser aplicada ao músculo durante o alongamento.

De acordo com a literatura, a intensidade de tensão no alongamento deveria ser aplicada até o sujeito referir um "incômodo”, “desconforto”, "tensão sem dor”, "leve sensação de alongamento" ou até o terapeuta sentir uma "rigidez” ou "restrição ao movimento" ",2,3,4,5,6,7,8,9,10. Em um estudo envolvendo pessoas com lesão medular, Harvey et al. ${ }^{11}$ observaram que os fisioterapeutas tendem a aplicar diferentes intensidades de tensão durante o alongamento quando não são guiados pela sensação de tensão e de desconforto do paciente. Este estudo ressalta a importância da sensação informada pelo paciente durante o alongamento. Com o intuito de quantificar essa sensação subjetiva, Björklund, Hamberg e Crenshaw ${ }^{12}$ utilizaram uma escala de Borg (de 0 a 10) para classificar a sensação do individuo a cada repetição de alongamento. Em outro estudo, os participantes indicaram a existência e o nível de desconforto sentido através de uma Escala Analógica Visual com índices de zero a dez, indicando nenhum e máximo desconforto ${ }^{13}$. Wiemann e Hahn ${ }^{1}$ estudaram a relação entre o torque e a informação subjetiva durante o alongamento passivo e observaram que, para um programa de curta duração, o efeito do alongamento passivo no ganho de amplitude de movimento (ADM) deve ser atribuído ao aumento da tolerância do indivíduo ao desconforto.

Como o limite de intensidade de tensão aplicada, encontrado nos estudos, é sempre determinado por informações subjetivas do paciente, nenhum autor conseguiu estabelecer uma intensidade adequada de estiramento em que o indivíduo deva permanecer para promover um alongamento efetivo. Na prática clínica, é freqüente a indagação sobre a quantidade de tensão que deve ser aplicada durante um alongamento em relação à sensação do paciente. Isso se deve, como já foi citado, à subjetividade das sensações e à diferença de sensibilidade entre cada indivíduo ${ }^{14,15}$.

Portanto, considerando que, de todas as variáveis empregadas no alongamento muscular, a intensidade de tensão a ser aplicada é a que apresenta maior dificuldade de aplicação, pretendeu-se, com esse trabalho, compreender melhor a relação entre tensão muscular e as informações subjetivas do indivíduo a respeito da sensação de desconforto gerada durante o alongamento.

\section{MATERIAIS E MÉTODOS}

\section{Voluntários}

Participam desse estudo 20 indivíduos, jovens, de ambos os sexos, sem história de patologias musculoesqueléticas, envolvendo o quadril e o joelho. Foram divididos aleatoriamente em dois grupos de 10 indivíduos, sendo: o grupo 1 (G1) formado de 5 homens e 5 mulheres (idade média de 21,3 $\pm 2,6)$ que foram submetidos a programa de alongamento muscular; e o grupo 2 (G2) formado de 5 homens e 5 mulheres (idade média de 19,6 $\pm 1,5$ ) sem programa de alongamento.

Os indivíduos selecionados não tinham experiência prévia em programas de alongamento muscular ou qualquer tipo de prática de alongamento e apresentavam, ao teste de flexibilidade, perda na extensão ativa do joelho maior que $30^{\circ}$ 2,3,4,5. Após a seleção, os voluntários assinaram Termo de Consentimento para a participação no experimento, aprovado pelo Comitê de Ética em Pesquisa da Universidade do Oeste Paulista (proc. 51/2003).

\section{Modelo experimental}

O grupo 1 (G1) foi submetido a um programa de alongamento dos músculos isquiotibiais no membro dominante, composto por três sessões semanais de alongamento durante 6 semanas consecutivas. Em cada sessão, foram realizados 3 alongamentos estáticos passivos de 30 segundos cada, com 10 segundos de intervalo entre cada alongamento ${ }^{4}$. Para a realização do alongamento, o voluntário era posicionado em decúbito dorsal sobre uma prancha de estabilização (com faixas de velcro presas nas laterais para fixação da pelve e do membro não dominante), sendo realizado o movimento de flexão coxofemoral com o joelho estendido. Os músculos foram mantidos alongados no momento em que o indivíduo relatava sensação de tensão de desconforto sem dor. O grupo 2 (G2) não praticou nenhum tipo de alongamento durante as seis semanas.

Na sétima semana, após o término do programa de alongamento do grupo 1, todos os voluntários foram submetidos a 3 avaliações, sendo duas no mesmo dia, com intervalo de 15 minutos entre elas (T1 e T2) e a terceira no 
dia seguinte (T3). Para a avaliação, utilizou-se o teste de alongamento dos músculos isquiotibiais, a partir do qual foi obtido o torque no momento de sensação de desconforto sem dor (SD) e no momento de sensação de desconforto com dor (SDD) e a atividade eletromiográfica (EMG) do músculo bíceps femoral. Para a realização do teste de alongamento, o voluntário era posicionado da mesma forma que a utilizada no programa de alongamento, com uma célula de carga acoplada a uma tornozeleira que foi fixada à perna dominante do voluntário e tracionada pelo terapeuta, num movimento de flexão coxofemoral lento e constante. Durante o movimento, o voluntário informava, apertando a pêra do esfignomanômetro, a posição em que sentia um "incômodo", "desconforto", "tensão sem dor” (SD) e, em seguida, a posição em que experimentava as mesmas sensações, porém com a presença de dor (SDD). Cada teste de alongamento foi repetido 3 vezes.

\section{Instrumentação}

Foi utilizado um sistema de aquisição de sinais composto por eletrodo bipolar ativo de superfície (pré-amplificado, com ganho de 20 vezes, modo de rejeição comum de $>80 \mathrm{~dB}$, impedância de 1012 Ohms // \%pF) para medida de EMG; célula de carga do tipo universal, com "strain gage”, modelo MM da Kratos Dinamômetros, com capacidade nominal para até $100 \mathrm{Kg}$, para medida da tensão de alongamento; eletrogoniômetro, constituído de duas hastes plásticas de $30 \mathrm{~cm}$ de comprimento, interligadas por um potenciômetro linear de $10 \mathrm{~K} \Omega$, para medida do ângulo da articulação coxofemoral; célula de carga sensível à pressão de ar (do tipo strain-gauge), acoplada a uma pêra de esfignomanômetro, utilizado para registrar a informação do paciente com relação à tensão de estiramento do músculo durante o alongamento (SD e SDD). O eletrodo, o eletrogoniômetro, a célula de carga e o sensor de pressão foram conectados a um módulo condicionador de sinais, modelo MCS 1000 - V2, da Lynx, com 16 canais de entrada. Nesse condicionador, os sinais analógicos de EMG eram armazenados e filtrados, utilizando um filtro passa-banda 20 e $500 \mathrm{~Hz}$ (Butterworth), amplificados para um ganho final de 1000 vezes e freqüência de $1000 \mathrm{~Hz}$ ${ }^{16,17}$. Esse sistema continha uma placa de conversão de sinal analógico para digital (A/D), modelo CAD 12/36 da Lynx, de 12 bites de resolução, com 16 entradas analógicas. O programa de aquisição de sinais AQDADOS, versão 5 da Lynx, serviu como software integrador do sistema de medidas dos sinais.

Para o cálculo do torque gerado pela ação passiva dos isquiotibiais, no sistema submetido ao alongamento, utilizou-se um modelo biomecânico bidimensional do sistema formado pelo quadril, joelho e tornozelo. A partir do diagrama e, considerando que o sistema se encontrava em equilíbrio estático, $\mathbf{S F}=0$ e $\mathbf{S} \tau=0$, em ambas as posições, $\mathrm{SD}$ e $\mathrm{SDD}$, obteve-se o torque passivo gerado pelos isquiotibiais através da equação $1^{1,18}$.
$\mathbf{T}_{\mathrm{FIT}}=\left(\mathbf{D}_{3} \cdot \mathbf{F}_{\mathbf{a}}\right)-\left(\mathbf{D}_{1} \cdot \mathbf{P}_{\mathbf{c}} \cdot \cos \boldsymbol{\theta}\right)-\left(\mathbf{D}_{2} \cdot \mathbf{P}_{\mathbf{p}} \cdot \cos \boldsymbol{\theta}\right)$ (equação 1)

onde:

$\mathrm{T}_{\text {FIT }}$ - torque gerado nos músculos isquiotibiais;

$\theta$ - ângulo de flexão da articulação do quadril;

$\mathrm{F}_{A}$ - força aplicada ao tornozelo do voluntário;

$\mathrm{D}_{1}$ - distância do eixo de rotação ao centro de gravidade da coxa;

$\mathrm{D}_{2}$ - distância do eixo de rotação ao centro de gravidade da perna;

$\mathrm{D}_{3}$ - distância do eixo de rotação ao maléolo lateral do tornozelo;

$\mathrm{P}_{c}$ - peso da coxa;

$\mathrm{P}_{p}$ - peso da perna;

$\mathrm{C}_{G C}$ - centro de gravidade da coxa;

$\mathrm{C}_{G P}$ - centro de gravidade da perna.

Os parâmetros da equação, acima descritos, podem ser observados no diagrama apresentado na figura 1. Para determinar o centro de gravidade de cada segmento do membro inferior, seus respectivos pesos e os braços de momento das forças envolvidas, utilizou-se modelo antropométrico adaptado de Zatsiorsky-Selvyanov ${ }^{18}$.

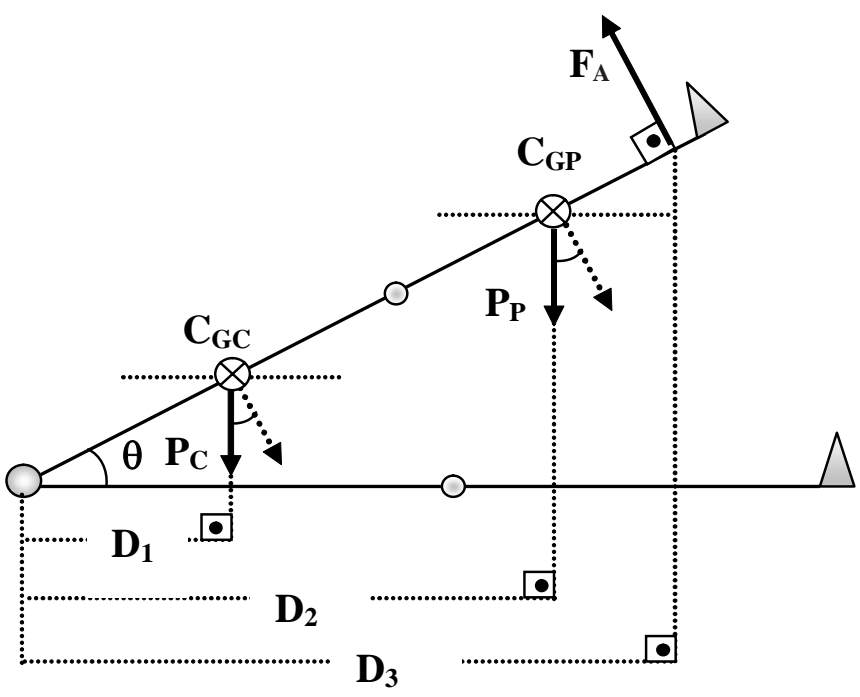

Figura 1. Diagrama representativo do modelo biomecânico bidimensional do sistema formado por quadril, joelho e tornozelo, mostrando a distribuição de forças que agem na coxa, na perna e seus ângulos de ação, que foram utilizados para o cálculo do torque gerado nos músculos isquiotibiais durante o teste de alongamento.

\section{Seleção dos dados para análise}

A figura 2 exemplifica os sinais coletados durante o teste de alongamento, mostrando o comportamento da atividade elétrica $(\mu \mathrm{V})$ do músculo bíceps femoral, da tensão muscular (Kgf) dos músculos isquiotibiais, do ângulo da articulação coxofemoral, gerado pelo eletrogoniômetro, e do sinal do sensor de pressão nos momentos de SD e de SDD. 


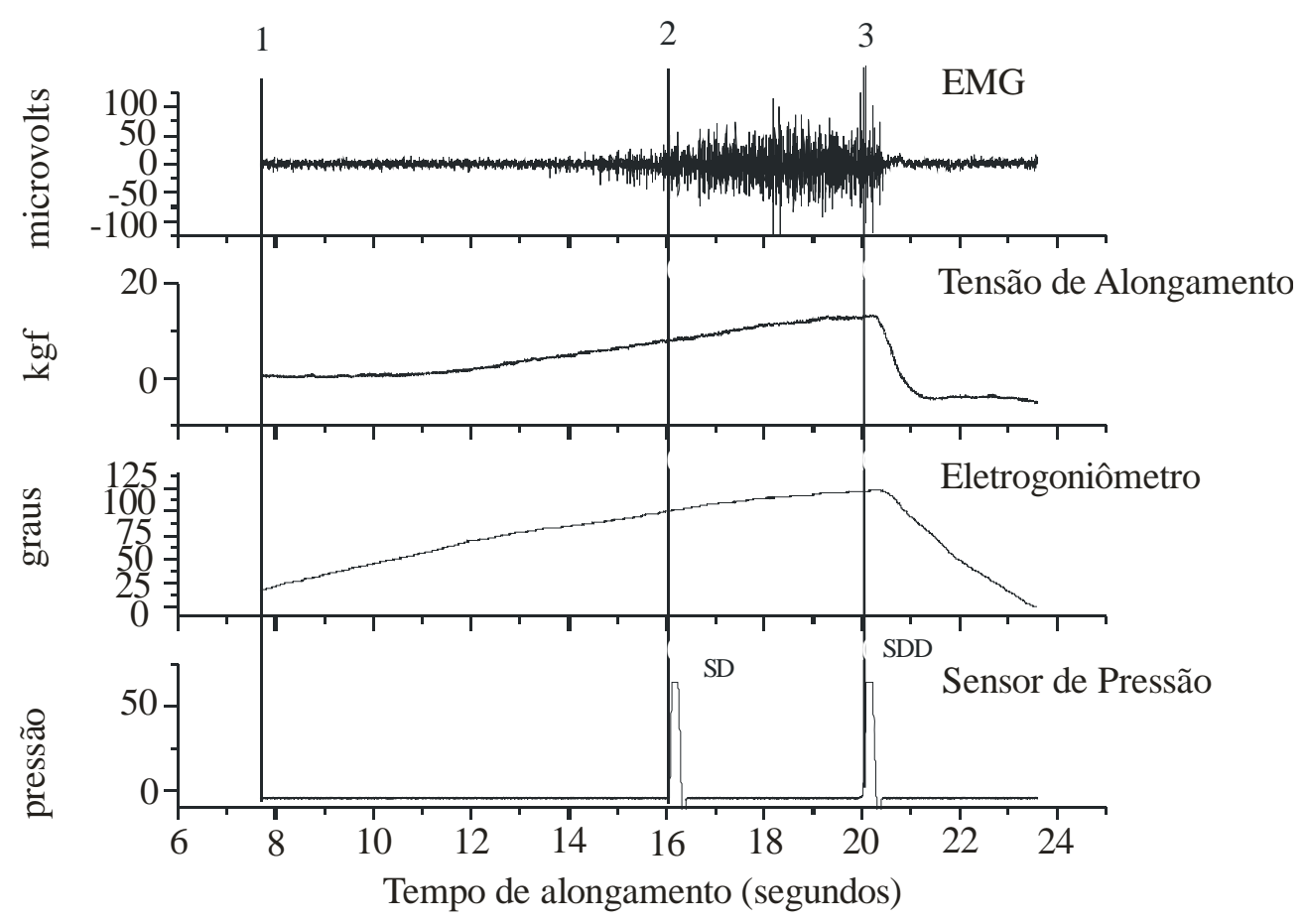

Figura 2. Exemplo de sinal coletado durante o teste de alongamento dos músculos isquiotibiais para avaliação das posições de sensação de desconforto (SD) sem dor e de sensação de desconforto com dor (SDD). Onde: (1) posição de repouso; (2) posição SD; (3) posição SDD.

Para a obtenção dos dados referentes a EMG, foram escolhidas três posições, representadas na Figura 2 pelas retas 1 , 2 e 3 que correspondem, respectivamente, ao repouso, a SD e a SDD. Os valores de EMG foram mensurados em RMS (root means square), em cada janela de $100 \mathrm{mseg}$. Para normalização do sinal EMG foi considerado o valor da contração isométrica voluntária máxima. Os valores de tensão de alongamento e do ângulo da articulação coxofemoral obtidos nos momentos de SD e SDD (retas 1 e 2) foram utilizados no cálculo para obtenção do torque muscular.

\section{Tratamento dos dados}

Para a análise dos valores de torque, foi utilizada a técnica da Análise de Variância Multipariada (MANOVA) para medidas repetidas em dois grupos independentes ${ }^{19}$. Já os valores de EMG normalizados foram analisados através da Técnica de Análise de Variância Não-Paramétrica para o modelo de medidas repetidas em dois grupos independentes ${ }^{20}$, com nível de significância de 95\%.

\section{RESULTADOS}

No início deste trabalho foi aplicado um programa de alongamento para o G1, cujos voluntários $(\mathrm{n}=10)$ apresentaram graus de encurtamento dos músculos isquiotibiais maior que $30^{\circ}$, antes do programa. Após 6 semanas, todos os voluntários apresentaram ganho médio de flexibilidade muscular de $14,5^{\circ}( \pm 4,378)$.

\section{Análise do torque SD}

Observando a figura 3, verifica-se que G1 apresenta maiores valores de torque na SD do que o G2, nas três avaliações, cujas diferenças foram consideradas estatisticamente significantes, com valores calculados de $\mathrm{F}=34,87(\mathrm{P}<0,01), 39,39(\mathrm{P}<0,01)$ e 13,6 $(\mathrm{P}<0,01)$, respectivamente para T1, T2 e T3. Com relação à análise do torque SD, nas três avaliações em cada grupo, nota-se que não existiram diferenças estatisticamente significantes entre elas, com valores calculados de $\mathrm{F}=1,16(\mathrm{P}>0,05)$ e 2,42 (P > 0,05); respectivamente para G1 e G2. Portanto, embora exista diferença de torque entre os grupos G1 e G2, os voluntários de cada grupo informaram a sensação de desconforto sem dor com mesmo valor de torque nas três avaliações.

\section{Análise do torque SDD}

O resultado da análise da SDD, apresentado na figura 4, mostra que os valores de torque são maiores para o G1, cujas diferenças em relação ao G2 foram consideradas estatisticamente significantes nas avaliações T1 e T2, com valores calculados de $\mathrm{F}=5,72(\mathrm{P}<0,05)$ e 10,29 $(\mathrm{P}<0,01)$, respectivamente, e não significante na avaliação $\mathrm{T} 3$, com valor calculado de $\mathrm{F}=2,29(\mathrm{P}>0,05)$.

A análise do torque da SDD, nas três avaliações em cada grupo, mostra que não existiu diferença estatisticamente significante entre elas, com valores calculados de $\mathrm{F}=2,89$ $(\mathrm{P}>0,05)$ e 2,08 ( $>$ > 0,05), respectivamente para G1 e G2. 
Dessa forma, verifica-se que os voluntários de cada grupo informaram a sensação de desconforto sem dor com o mesmo valor de torque nas três avaliações, embora exista diferença nos valores de torque entre os grupos nas duas primeiras avaliações.

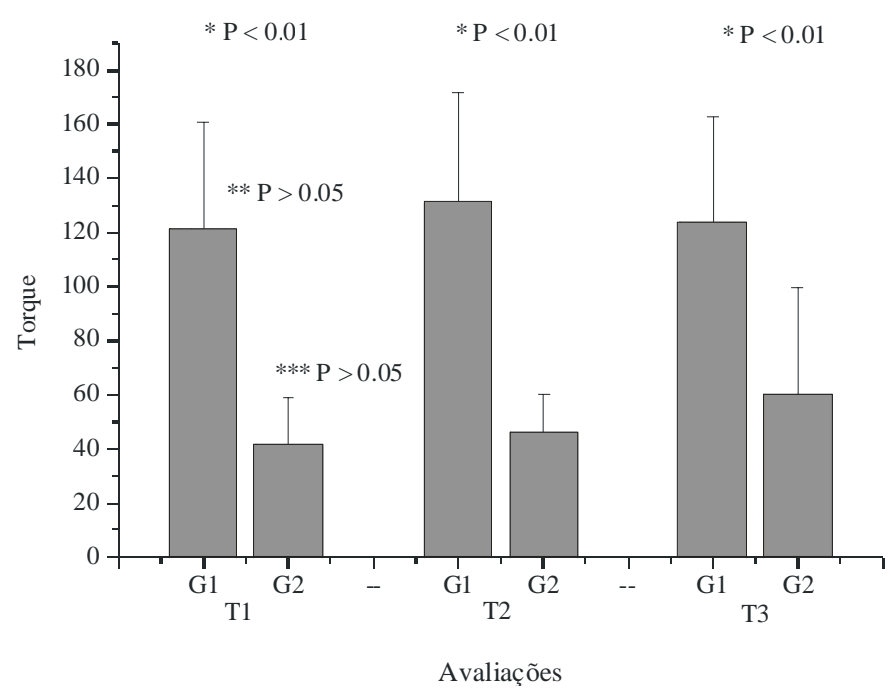

* = diferença significante entre G1 e G2; ** = diferença não significante entre T1, T2 е T3 no G1; *** = diferença não significante entre T1, T2 e T3 no G2.

Figura 3. Valores médios e desvios-padrão do torque calculado na posição da sensação de desconforto sem dor (SD), nas três avaliações (T1, T2 e T3) para os grupos G1 $(n=10)$ e G2 $(n=10)$, obtidos durante teste de alongamento dos músculos isquiotibiais.

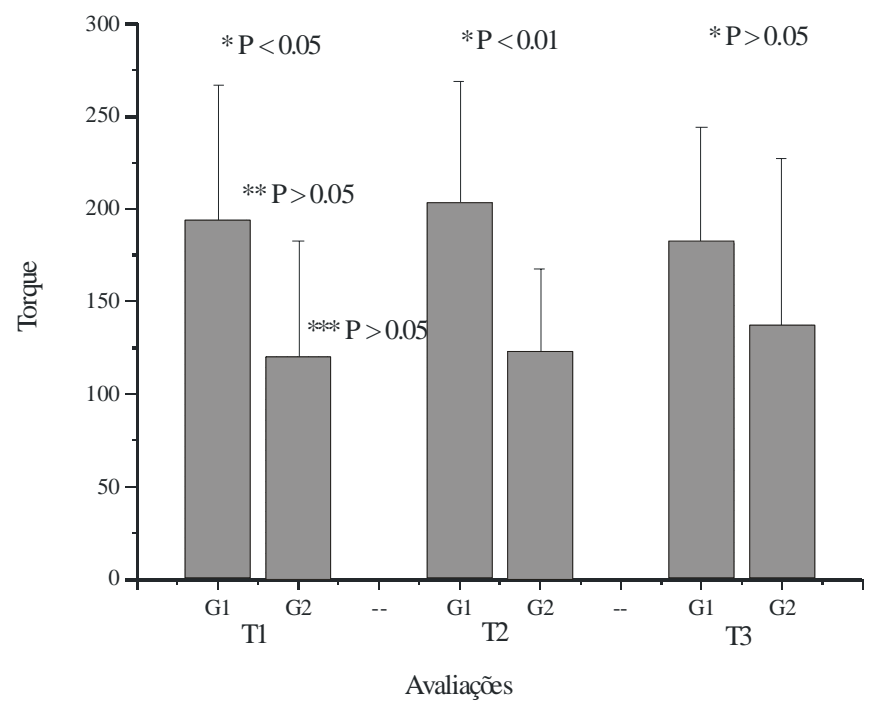

* = diferença significante entre G1 e G2 em T1 e T2; e não significante em T3; ** = diferença não significante entre T1, T2 e T3 no G1; *** = diferença não significante entre T1, T2 е T3 no G2.

Figura 4. Valores médios e desvios-padrão do torque calculado na posição de sensação de desconforto com dor (SDD), nas três avaliações (T1, T2 e T3) para os grupos G1 $(n=10)$ e G2 $(n=10)$, obtidos durante teste de alongamento dos músculos isquiotibiais.

\section{Análise da EMG na SD e na SDD}

As diferenças entre os valores da porcentagem de EMG demonstram que não existiu diferença estatisticamente significante entre a atividade de repouso SD e SDD, tanto para os dois grupos como para as três avaliações de cada grupo ( $\mathrm{P}>0,05)$ (Tabela 1 e 2). Portanto, como os valores de EMG dos músculos bíceps femoral, captados por meio de eletromiografia de superfície não apresentam diferenças, verificou-se que, durante o teste de alongamento, não foi captada atividade elétrica pelos eletrodos.

\section{DISCUSSÃO}

Os resultados da análise estatística mostraram que, no momento SD, embora os valores de torque revelem haver diferença estatística significante entre os grupos (o que era esperado em função do maior ângulo de flexibilidade permitido e da maior força de tração aplicada no grupo submetido ao programa de alongamento - G1), os dois grupos estudados apresentaram perfis semelhantes em relação à informação subjetiva de sensação durante as avaliações. Os resultados deste estudo apontam que a informação de desconforto sem dor ao alongamento (SD), embora subjetiva, é um parâmetro individual confiável e reprodutível para determinação de quantidade de tensão a ser aplicada pelo terapeuta durante alongamento muscular. Cabe lembrar que, neste trabalho, a SD foi utilizada no programa de alongamento para o G1 e que, após 6 semanas de alongamento, resultou em aumento na flexibilidade dos músculos isquiotibiais ganho médio de $14,5^{\circ}$. Os resultados encontrados neste trabalho estão de acordo com Bandy, Irion e Briggler ${ }^{4,5}$ e Sullivan, Dejulia e Worre $^{2}$, que também utilizaram como limite de intensidade de alongamento a sensação de desconforto sem dor para análise dos efeitos do tempo e da freqüência do alongamento estático.

Com relação ao comportamento dos valores de torque nas três avaliações de cada grupo, tanto para SD (Figura 3) como para SDD (Figura 4), observa-se que para o G1 os valores médios de torque em T2 foram maiores do que em T1 e T3; já para o G2, os valores médios foram crescentes nas três avaliações. Embora o comportamento do G1 não tenha sido relevante estatisticamente, pode-se inferir que o maior valor da média de torque, na segunda avaliação (T2), seja devido à adaptação do indivíduo à sensação de alongamento, possivelmente como resultado do efeito adaptativo do fuso muscular às manobras de alongamento, uma vez que o teste de alongamento desta avaliação foi realizado apenas 15 minutos após o teste da primeira. O mesmo comportamento não ocorreu no G2 possivelmente devido ao despreparo dos indivíduos quanto à sensação de alongamento. Essa possível adaptação sensorial reforça os achados de Björklund, Hamberg e Crenshaw ${ }^{12}$, que sugerem a antecedência da mesma à mudança na tenacidade muscular.

Os resultados da análise para a posição SDD revelaram que a análise comparativa dos valores de torque das avaliações 
Tabela 1. Valores da mediana e semi-amplitude da porcentagem de ativação eletromiográfica do músculo bíceps femoral nas condições de repouso, sensação de desconforto sem dor (SD) e sensação de desconforto com dor (SDD), nas três avaliações (T1, T2 e T3) dos testes de alongamento do grupo 1.

\begin{tabular}{|c|c|c|c|c|}
\hline G1 & \multicolumn{3}{|c|}{ Testes de alongamento } & \multirow{2}{*}{ Resultado do teste } \\
\hline Condições & T1 & $T 2$ & T3 & \\
\hline Repouso & $14,00 \pm 18,80$ & $12,25 \pm 17,12$ & $10,55 \pm 30,05$ & $P>0,05$ \\
\hline$S D$ & $13,75 \pm 18,55$ & $13,20 \pm 16,70$ & $10,75 \pm 28,95$ & $P>0,05$ \\
\hline$S D D$ & $18,20 \pm 16,60$ & $14,75 \pm 18,15$ & $13,60 \pm 19,60$ & $P>0,05$ \\
\hline Resultado do teste & $p>0,05$ & $p>0,05$ & $P>0,05$ & \\
\hline
\end{tabular}

Tabela 2. Valores da mediana e semi-amplitude da porcentagem de ativação eletromiográfica do músculo bíceps femoral nas condições de repouso, sensação de desconforto sem dor (SD) e sensação de desconforto com dor (SDD), nas três avaliações (T1, T2 e T3) dos testes de alongamento do grupo 2.

\begin{tabular}{ccccc}
\hline G2 & \multicolumn{3}{c}{ Testes de alongamento } & Resultado do teste \\
Condições & $T 1$ & $T 2$ & $T 3$ & \\
\hline Repouso & $6,25 \pm 5,95$ & $6,35 \pm 5,70$ & $6,00 \pm 5,80$ & $\mathbf{P}>\mathbf{0 , 0 5}$ \\
SD & $7,35 \pm 6,60$ & $6,60 \pm 9,75$ & $5,50 \pm 8,15$ & $\mathbf{P}>\mathbf{0 , 0 5}$ \\
SDP & $8,40 \pm 17,90$ & $7,50 \pm 18,10$ & $6,55 \pm 20,70$ & $\mathbf{P}>\mathbf{0 , 0 5}$ \\
Resultado do teste & $\mathbf{p}>\mathbf{0 , 0 5}$ & $\mathbf{p}>\mathbf{0 , 0 5}$ & $\mathbf{P}>\mathbf{0 , 0 5}$ & \\
\hline
\end{tabular}

nos grupos G1 e G2 demonstram haver diferença estatística significante, com o primeiro grupo apresentando valores de torque maiores que o segundo em razão da maior flexibilidade obtida durante o programa de alongamento. Entretanto, em relação à informação de sensação subjetiva, durante a avaliação de alongamento no momento $\mathrm{T} 3$, as informações de sensações subjetivas foram estatisticamente semelhantes entre os grupos. Observando os resultados, nota-se que a média dos valores de torque para o G1, no momento SDD da terceira avaliação, diminuiu, enquanto a média dos valores de torque, no momento SDD para o G2, aumentou nesta mesma avaliação.

Considerando que os mecanoceptores e proprioceptores musculares e articulares são, em grande parte, responsáveis pelo aumento da tolerância ao alongamento por apresentarem redução de disparo após uma única manobra de alongamento ${ }^{12}$, a redução da SDD para o G1 pode indicar que, um intervalo de 24 horas entre um alongamento e outro é tempo suficiente para que os receptores musculares e articulares de um indivíduo sejam novamente estimulados. No entanto, o mesmo comportamento não se observa no G2 que, ao contrário do esperado, apresentou maiores valores de torque. Porém, uma análise mais detalhada dos valores de torque de cada indivíduo do G2, em relação às avaliações realizadas, revela que os crescentes valores de torque não estariam relacionados ao aumento da tolerância do indivíduo em relação ao alongamento e sim ao despreparo desses em informar a sensação subjetiva durante o alongamento, resultando assim em alto valor de desvio-padrão. Portanto, na terceira avaliação, a semelhança entre os valores de torque obtidos para a posição SDD pode ser relacionada com a redução da tensão de dor suportada pelo G1, associada ao aumento do valor médio de torque do G2 em razão do alto desvio-padrão ocorrido (sd \pm 90,17).

Com relação ao comportamento dos valores de torque entre as três avaliações dentro de um mesmo grupo, não se observam diferenças estatisticamente significantes, mostrando que as informações subjetivas de sensação ao alongamento da SDD se reproduziram nos momentos testados (T1, T2, T3). Desta forma, compreende-se que a informação de desconforto com dor ao alongamento (SDD), semelhante ao SD, também é um parâmetro reprodutível.

Harvey et al. ${ }^{11}$, sugerem que indivíduos com sensibilidade normal toleram torques de alongamento dos músculos isquiotibiais entre $20 \mathrm{Nm}$ e $60 \mathrm{Nm}$. Neste estudo, os valores de torque para o momento de tensão de desconforto 
observados variam entre $49 \mathrm{Nm}$ e 197,4 Nm para o grupo 1 (média $=125,46, \pm 39,43$ ) e de 19,4 a $138 \mathrm{Nm}$ para o grupo 2 (média $=50,62 \pm 23,73$ ), o que indica que indivíduos acostumados à prática de alongamento tendem a suportar maiores valores de torque e, ainda que alguns estudos mostrem que torque de alongamento dos músculos isquiotibiais seja entre $20 \mathrm{Nm}$ e $60 \mathrm{Nm}$, os nossos dados revelam que, para indivíduos treinados, é possível aplicar torques acima de 60 Nm sem riscos de provocar lesão muscular. Halbertsma et al. ${ }^{8}$, Wiemann e Hahn ${ }^{1}$ e Magnusson et al. ${ }^{9}$ utilizaram como parâmetro de intensidade o momento de sensação desagradável de alongamento muscular indicado pelo voluntário, também descrita como sendo a primeira sensação de dor, e não relatando caso de lesão muscular durante a realização do programa de alongamento. Este trabalho sugere que intensidade ideal de se aplicar o alongamento passivo seria um pouco além da tensão de desconforto, uma vez que o intervalo encontrado entre essa tensão e o aparecimento da dor é amplo e seguro o suficiente para que se possa utilizálo durante o alongamento e maximizar o ganho de ADM.

As análises dos resultados da atividade eletromiográfica não revelaram diferenças estatisticamente significantes entre os dois grupos nas diferentes avaliações testadas (T1, T2, T3). O baixo grau de atividade eletromiográfica encontrado reflete a capacidade de relaxamento do sistema neuromuscular ${ }^{1}$, uma vez que todos os indivíduos foram instruídos a não resistirem ativamente durante o alongamento. Osterning et al. ${ }^{10}$ sugerem que, quando o alongamento estático é contínuo, por um longo período, sinais inibitórios eventualmente superam os impulsos excitatórios resultantes da tensão de alongamento e dessa forma proporcionam o relaxamento. Os resultados deste estudo estão de acordo com a bibliografia encontrada e revelam que a atividade eletromiográfica presente durante o alongamento muscular é muito baixa ${ }^{13,1,8,9}$.

\section{CONCLUSÃO}

Observou-se com este trabalho que a informação do indivíduo em relação à sensação subjetiva de alongamento é confiável, reprodutível e eficiente, quando o alongamento é realizado no limite de tensão de desconforto sem dor. $\mathrm{O}$ intervalo existente entre a tensão de desconforto sem dor e o surgimento da dor é amplo o suficiente para permitir que o alongamento seja realizado entre estes dois limites de tensão, visando maximizar o ganho de flexibilidade.

Os valores de EMG do músculo bíceps femoral, captados por meio de eletromiografia de superfície, não apresentaram diferenças, demonstrando que durante os testes de alongamento não foi captada atividade elétrica pelos eletrodos.

Agradecimentos: FAPESP - Processo n 03/12239-6.

\section{REFERÊNCIAS BIBLIOGRÁFICAS}

1. Wiemann K, Hahn K. Influences of strength, stretching and circulatory exercises on flexibility parameters of human hamstring. Int J Sports Med. 1977; (18): 340-6.

2. Sullivan MK, Dejulia JJ, Worrell TW. Effect of pelvic position and stretching method on hamstring muscle flexibility. Medicine and Science in Sports and Exercise. 1992; 1383-9.

3. Bandy WD, Irion JM. The effect of time on static stretching on the flexibility of hamstring muscle. Physical Therapy. 1994; 74(9): 845-852.

4. Bandy WD, Irion JM, Briggler M. The effect of time and frequency of static stretching on flexibility of the hamstring muscles. Physical Therapy. 1997; 77(10): 1090-6.

5. Bandy WD, Irion JM, Briggler M. The effect of static stretch and dynamic range of motion on the flexibility of the hamstring muscles. JOSPT. 1998; 27(4): 295-300.

6. Sady SP, Wortman M, Blanke D. Flexibility training: ballistic, static or proprioceptive neuromuscular facilitation? Archives of Physical Medicine and Rehabilitation. 1982; (63): 261-3.

7. Feland JB, Myrer JW, Schulthies SS, Fellingham GW, Measom GW. The effect of duration of stretching of the hamstring muscle group for increasing range of motion in people aged 65 years or older, Physical Therapy. 2001; 81(5): 1110-7.

8. Halbertsma JPK, Mulder I, Goeken LNH, Eisma WH. Repeated passive stretching: acute effect on the passive muscle moment and extensibility of short hamstrings. Archives of Physical Medicine and Rehabilitation. 1999; 80(4): 407-414.

9. Magnusson SP, Simonsen EB, Aagaard P, Sorensen H, Kjaer M. A Mechanism for altered flexibility in human skeletal muscle. Journal of Physiology. 1996; (497): 291-8.

10. Osterning LR. Muscle activation during proprioceptive neuromuscular facilitation (PNF) stretching techniques. American Journal of Physical Medicine. 1987; 66(5): 298-307.

11. Harvey LA, McQuade L, Hawthorne S, Byak A. Quantifying the magnitude of torque physiotherapist apply when stretching the hamstring muscle of people with spinal cord injury. Archives of Physical Medicine and Rehabilitation. 2003; 84(7): 1072-5.

12. Björklund M, Hamberg J, Crenshaw AG. Sensory adaptation after a 2-week stretching regimen of the rectus femoris muscle. Archives of Physical Medicine and Rehabilitation. 2001; (82): 1245-9.

13. Azevedo CCF, Ruzzante DM, Loss JF, Martinez F. Resposta eletromiográfica durante alongamento passivo estático: uma analise preliminar. X Congresso Brasileiro de Biomecânica 2003; Fev.

14. Achour Jr A. A flexibilidade: teoria e prática. Atividade Física \& Saúde, 1998.

15. Alter MJ. Ciência da flexibilidade. $2^{\mathrm{a}}$ ed. Porto Alegre: Artes Médicas Sul. 1999.

16. Acierno SP, Baratta RV, Solomonow M. A practical guide to electromyography for biomechanics. Louisiana State University, 1995.

17. De Luca. The use of Surface Electromyography in Biomechanics. Journal of Applied Biomechanics. 1997; (13): 135-163. 
18. Leva P. Adjustments to Zatsiorsky - Selvyanov‘s segment inertia parameters. Journal of Biomechanics. 1996; 29(9): $1223-1230$.

19. Johnson RA, Wichern DW. Applied Multivariate Statistical Analysis. 4a ed. New Jersey: Prentice-Hall, New Jersey; 1998.
20. Norman GR, Streiner DL. Biostatistics - The base essential. St. Louis: Mosby Year Book; 1994. 